\title{
A Probable Connection between Solar and Atmospheric Decadal Variability
}

\author{
By KARIN LABITZKE ${ }^{1}$ AND HARRY VAN LOON \\ ${ }^{1}$ Freie Universität, Berlin, Germany \\ ${ }^{2}$ National Center for Atmospheric Research, Boulder, CO 80307, USA
}

The paper reviews briefly some recent investigations of possible effects of decadal solar variations on the stratosphere and troposphere, drawing attention to the stable, strong correlations between the height of constant pressure levels in the stratosphere and the sunspot cycle.

\section{Introduction}

The growing interest in the variability of climate has sharpened the realization that our knowledge of the natural variability and forcing of the atmosphere is imperfect. Partly as a result of the restricted insight into the causes of climate change, interest has revived in the possible effects of the Sun's variability on atmospheric variations. The popular belief of the past century was - to paraphrase Godson (1991) - that the variable solar activity had no influence on climate, or that if it did, the effects should always be the same with regard to sign and size. This scepticism is largely due to the fact that the statistical investigations, which dominate the research, although they often produce suggestive results, have been limited by their failure to explain how the Sun could produce the observed variations in the atmosphere. Some of the recently published papers reviewed below are no exception in this respect, but the statistics they present are of sufficient significance that they should be taken into account - even if their underlying cause should turn out not to be solar.

\section{Effects of the 11-year sunspot cycle}

\subsection{Summer}

If the 11-year solar cycle has a noticeable effect on the Earth's atmosphere, there is no self-evident reason why the outcome should be the same everywhere, or that it should appear as a purely radiative effect. The statistics presented below (based on the historical daily analyses at the Freie Universität, Berlin) suggest that dynamics play a major role in the spatial distribution of the atmosphere's response to the 11-year solar variability. This is evident in Figure 1, which shows the correlation between a 36-year series of $30-\mathrm{mb}$ height in summer and the $10.7 \mathrm{~cm}$ solar flux. This flux is a good index of the sunspot cycle. We have computed such correlations every year during the past five years, starting with a sample of 31 , and they have remained stable with each additional year. The correlations over more than three quarters of the area are locally significant above the $1 \%$ level; and over one third of the area the local significance is above the $0.1 \%$ level. The map as a whole is field significant at better than the $1 \%$ level (see Livezey \& Chen (1983), for an explanation of field significance). The shape of the correlation pattern, which is repeated in each month of the year, implies that the probable influence of the 11-year solar cycle is areally concentrated by atmospheric motion's.

In order for the $30-\mathrm{mb}$ geopotential height to vary with a period similar to that of the 11-year sunspot cycle, as suggested by Figure 1, the temperature of the air column below or the surface pressure, or both, must contain the same periodicity. As shown 


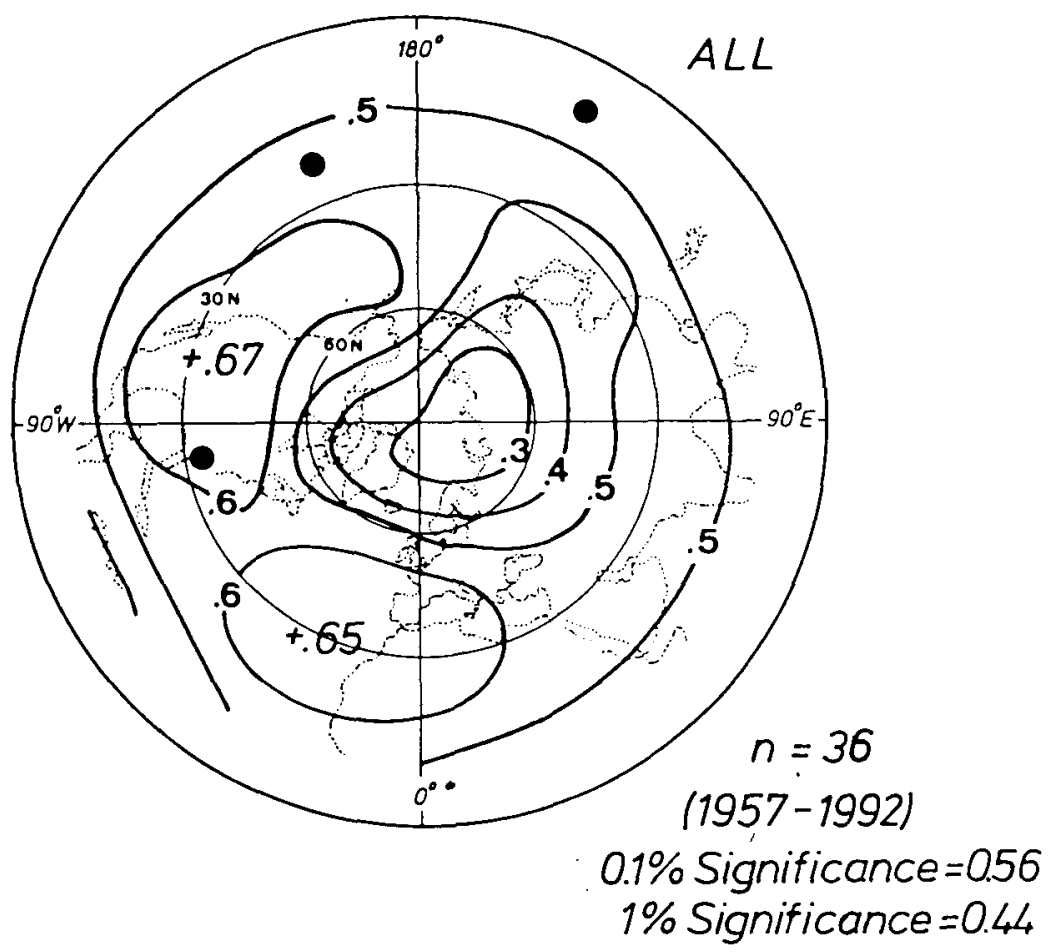

Figure 1. Correlations between $30-\mathrm{mb}$ height in July-August and the $10.7 \mathrm{~cm}$ solar flux. The $0.1 \%$ local significance level is 0.56 . The dots mark the locations in Figure 2. Updated from van Loon \& Labitzke (1990).

in the following, this periodicity does not exist in the surface pressure but is obvious in the temperature: Figures 2a,b show the difference in the temperature between solar maxima and minima from the ground to $30 \mathrm{mb}$ (about $24 \mathrm{~km}$ ) at two lower-latitude stations in areas of Figure 1 where the correlations are high. The two have in common that the troposphere is warmer in solar maximum, that the difference is negative in the levels about the tropopause $(100 \mathrm{mb})$, and that on average the air in the stratosphere is warmer in the solar maxima. At the station nearest the axis of highest correlation, Charleston, the average temperature difference increases with height above $700 \mathrm{mb}$ to $1.5^{\circ} \mathrm{C}$ just below the tropopause, at Truk the largest average difference is $0.8^{\circ} \mathrm{C}$. The association between the 11-year solar cycle and the global temperature in the upper troposphere and lower stratosphere was studied by Sellers \& Liu (1988). They found that the low frequency variation of the first EOF in the temperature is "primarily and strongly" correlated with the quasi-decadal solar cycle.

These correlations in the vertical do not prove that a solar influence exists which affects the stratosphere through the troposphere, either directly in the latitudes concerned or indirectly from low latitudes through the Hadley circulation. It is, for instance, also possible that the stratosphere warms at solar maximum through absorption of UV radiation by ozone (Hood et al. 1993), and that this changes the vertical distribution of temperature and thereby changes the height and temperature of the tropopause. The temperature in the upper troposphere would be affected in this way. Another mechanism 

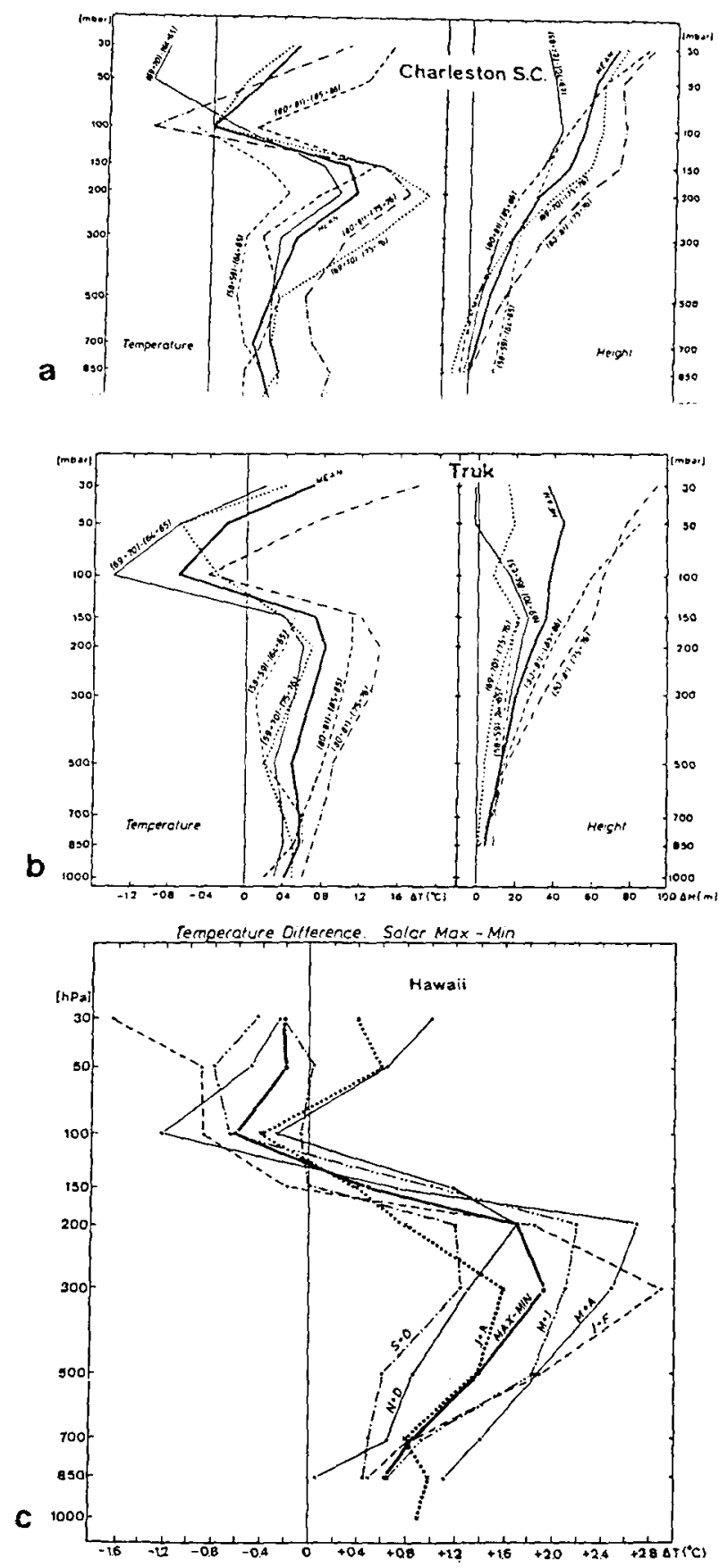

FIGURE 2. The change of temperature and geopotential height in July-August from solar minimum to maximum at Charleston (South Carolina) and Truk $(7.5 \mathrm{~N}, 152 \mathrm{E})$. The heavy line is the average. From Labitzke \& van Loon (1992). (c) The average temperature change from solar minimum to maximum at Lihue $(22 \mathrm{~N}, 159 \mathrm{~W})$, through the year and in the annual mean (heavy solid line). From Labitzke \& van Loon (1993). 
is that of forcing atmospheric changes due to variations in latent heat release from effects on cloud microphysics of solar wind induced changes in the global electric circuit (Tinsley \& Deen 1991).

A more plausible idea is that the Sun at its highest 11-year activity heats the ocean surface at low latitudes more than at its lowest activity. This would intensify the Hadley circulation in solar maxima, causing warming in the subtropics by increased subsidence and thus affect the tropopause and the lower stratosphere. The objection to this idea is that the change of total solar irradiance from solar minimum to maximum, as measured by satellite during the recent solar cycle, amounts to only $0.1 \%$. The warming of the tropical water in the solar maximum need not be large: the drastic change in the global atmospheric circulation in the years after 1977 (Labitzke \& van Loon 1993) was accompanied by an average change in the tropical sea surface temperature of only $0.3^{\circ} \mathrm{C}$ (Bottomley et al. 1990), which is not far from observational accuracy.

\subsection{Midwinter}

The basic shape of the correlation pattern between $30-\mathrm{mb}$ height and the 11-year solar cycle, a crescent of high correlations along $30^{\circ} \mathrm{N}$ on the water-dominated side of the hemisphere, is also present in January-February (Figure 3a) but the correlations are lower than in the 10 other months of the year (cf., Figure 1), reaching the $5 \%$ significance level only between $20^{\circ} \mathrm{N}$ and $45^{\circ} \mathrm{N}$ over the Pacific Ocean. It was found, however, that the stratosphere responds differently to solar changes in either extreme of the Quasi-Biennial Oscillation (QBO) in the 50-40 mb wind of the equatorial stratosphere (Labitzke 1987). In the east years (Figure $3 \mathrm{~b}$ ) the correlation pattern is the same as in the ten other months of the year, and over more than half of the map the correlations are locally significant above the $5 \%$ level. If one assumes that the solar effect expresses itself through changes in the atmosphere where the crescent of high correlations lies, then - owing to the marked teleconnections in winter (Shea et al. 1992) - the changes at high latitudes must be opposite to those in the tropics and subtropics and thus account for the large negative correlations in the Arctic (Figure 3b). In the east years of the QBO, the polar vortex tends, in other words, to be stronger at high and weaker at low solar activity, whereas theory (Holton \& Tan 1980, 1982) and model experiment (e.g., (Dameris \& Ebel 1990) indicate that the vortex should generally be weak in east years.

This relationship also emerges from the scatter diagram in Figure 4: the correlation between the zonally-averaged geostrophic wind at $60^{\circ} \mathrm{N}$ in the east years and the solar flux is 0.7 , which is at the $1 \%$ significance level. The asterisks in the diagram mark the winters when major midwinter warmings (breakdowns of the polar vortex) took place in January or February; they are prevalently associated with lower values of the solar flux.

Conditions are different in the west years of the QBO (Figure 3c): in these years there are no statistically significant correlations over most of the map, the only noteworthy ones (positive) being over the Arctic, and there is no sign of the strong teleconnections between higher and lower latitudes which otherwise dominate the winter climate of the stratosphere (Shea et al. 1992). In the west years the Arctic stratosphere is positively correlated with the solar cycle (see the scatter diagram of north polar, 30-mb temperature versus the $10.7 \mathrm{~cm}$ flux in Figure 5) because the major midwinter warmings in these years take place only at solar maximum.

Our correlations suggest that high solar activity brings on a disturbed state: at solar minimum the vortex is intense in the west and weak in the east years which, as mentioned above, is predicted by theory and confirmed by experiment; in contrast, at solar maximum the vortex weakens in the west and strengthens in the east years, contrary to theory. 

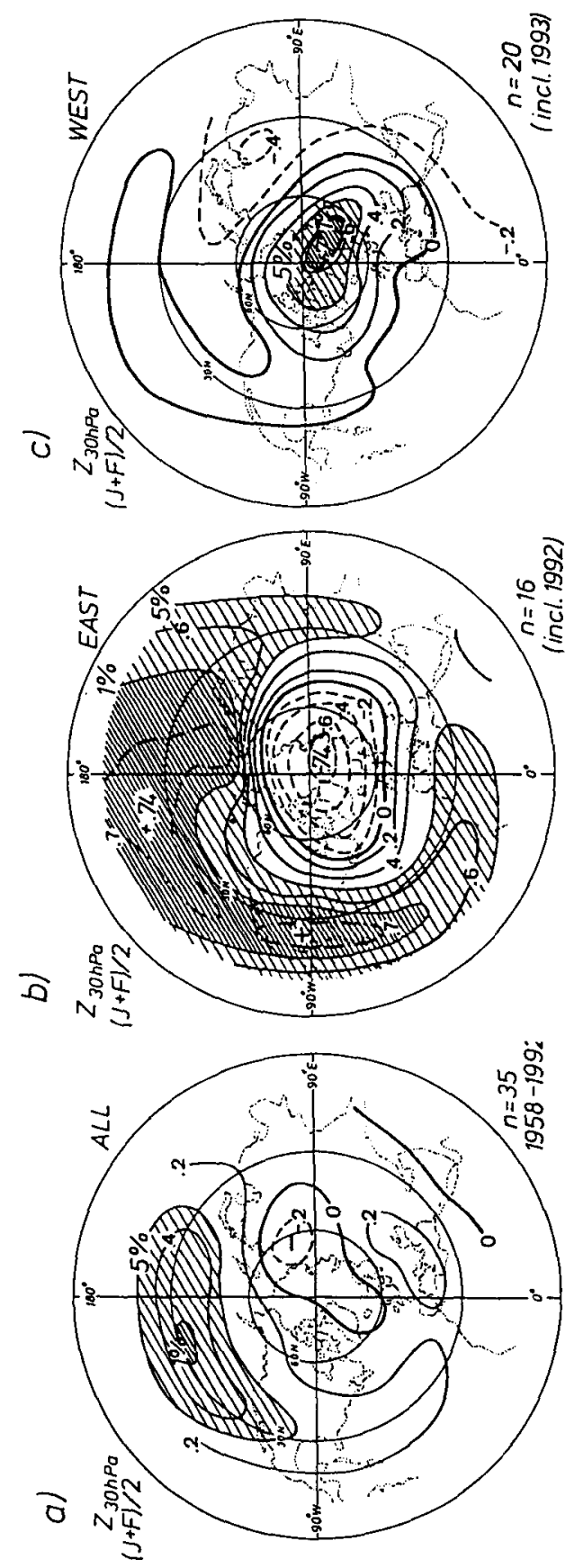

Figure 3. Correlations between the $10.7 \mathrm{~cm}$ solar flux and (a) the $30-\mathrm{mb}$ height in January-February, $n=35$ years; (b) in the east years of the QBO; (c) in the west years. The areas where the local statistical significance is above the $5 \%$ level are lightly hatched, those above the $1 \%$ level are densely hatched. Updated from Labitzke \& van Loon (1988). 


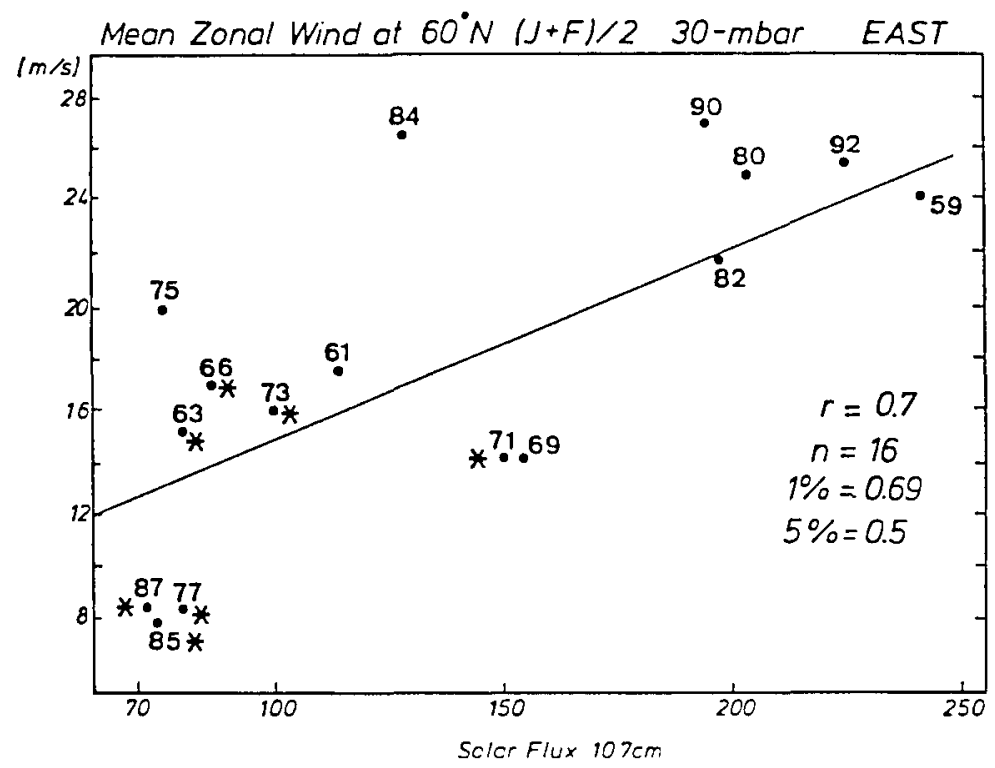

FiguRE 4. Zonally-averaged geostrophic wind at $60 \mathrm{~N}$ in January-February at $30-\mathrm{mb}$ in the east years plotted against the $10.7 \mathrm{~cm}$ solar flux. The asterisks denote major midwinter warmings.

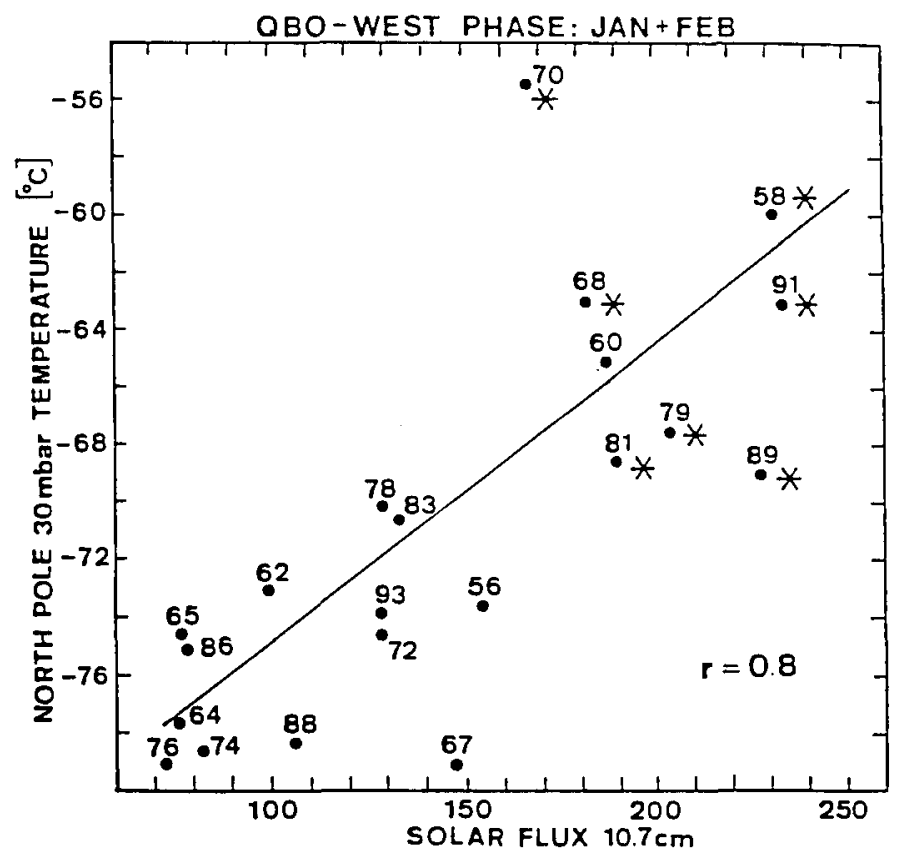

Figure 5. 30-mb temperature at the North Pole in January-February plotted against the $10.7 \mathrm{~cm}$ solar flux in the west years of the QBO. Asterisks denote major midwinter warmings. Updated from van Loon \& Labitzke (1990). 
ANNUAL, 1958-1992, $r\left(Z_{30}, 10.7 \mathrm{~cm}\right.$ FLUX)

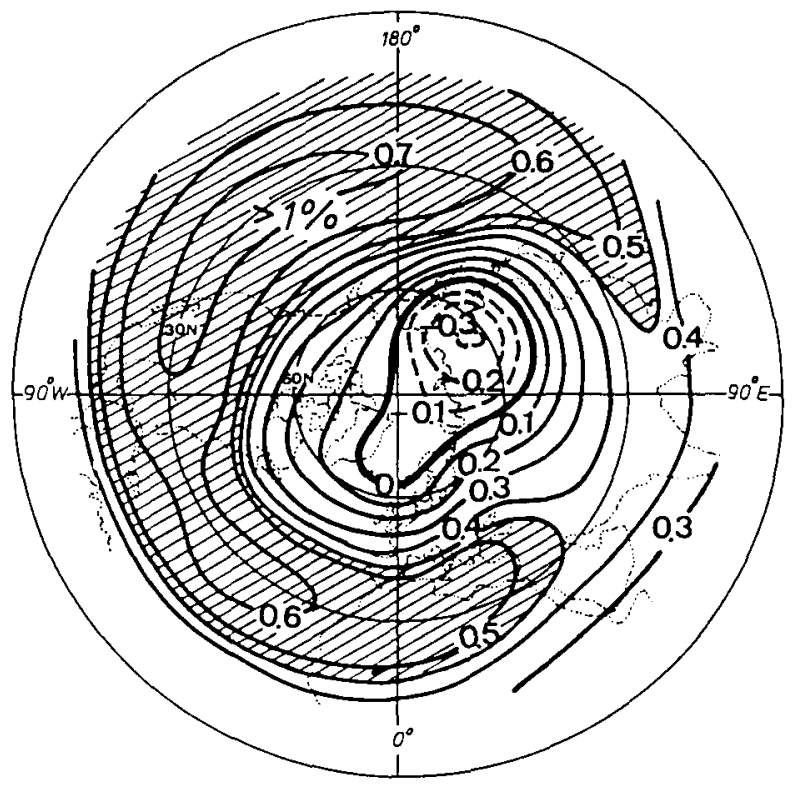

Figure 6. Correlations between the annual mean 30-mb height, 1958-1992, and the $10.7 \mathrm{~cm}$ solar flux. In the hatched areas the local statistical significance exceeds the $1 \%$ level. Updated from van Loon \& Labitzke (1990).

\subsection{Year}

The map of correlation between the annual mean $30-\mathrm{mb}$ height and the $10.7 \mathrm{~cm}$ solar flux (Figure 6) sums up the statistical analyses of this section. Not unexpectedly, the pattern is that of a crescent of strong, stable correlations. The latter exceed the $1 \%$ significance level over half the area of the map, and are above the $0.1 \%$ significance level from $150^{\circ} \mathrm{E}$ westward to $10^{\circ} \mathrm{W}$, between $15^{\circ}-20^{\circ} \mathrm{N}$ and $40^{\circ}-45^{\circ} \mathrm{N}$. Even though no physical mechanism has yet been proposed to explain the large and stable statistical association between the 11-year solar cycle and the changes in the lower stratosphere, it is reasonable to propose that correlations such as those in Figures 6 and 1 are not due to chance. It is also unlikely that the decadal variation which they reflect is due to an internal oscillation in the ocean-atmosphere system with a period close to that of the solar cycle, since it is unlikely that precisely in the time interval which was available to us such an internal oscillation should have been in phase with the solar cycle.

The crescent of high positive correlations in Figure 6 indicates that the atmosphere below the $30-\mathrm{mb}$ level must in the annual mean be warmer at the peaks than in the valleys of the 11-year solar cycle. This is illustrated in Figure 2c for Hawaii where the correlation is between 0.6 and 0.7 in Figure 6: the heavy solid line is the mean annual temperature difference between maxima and minima in three solar cycles. The difference is positive in the troposphere, reaching almost two degrees at $300 \mathrm{mb}$ to $200 \mathrm{mb}$, but slightly negative above the tropopause. The large positive correlation coefficient between the 30-mb height and the solar flux is thus due entirely to the difference in the tropospheric temperature between the extremes of the 11-year sunspot cycle. 
The vertical structure of the temperature difference has the same shape throughout the year, but the values are smaller in summer and autumn than in winter and spring (Figure 2c). Evidently, the effect therefore is not directly linked with the local elevation of the Sun but must be associated with the interseasonal changes of the general circulation. The highest correlations in Figure 6 are in the belt of predominantly downward motion in the subtropical high; and the higher mean temperature in the solar maxima than minima is thus likely associated with differences in the strength of the subsidence. The subsidence is stronger in winter and spring than in summer and autumn because the convergence in the upper troposphere between the meridional flow in the Hadley and Ferrell circulations is stronger during the winter and spring when the temperature difference between equator and the North Pole is largest.

\section{Conclusion}

Satellites have in recent years provided a reliable picture of changes in the Sun's irradiance during a sunspot cycle; and papers have appeared with statistics which imply that the lower atmosphere responds to solar variability on several time scales. On the decadal time scale, the correlations of Labitzke \& van Loon (1988), (1992) and van Loon \& Labitzke (1990) have remained stable and increasingly statistically significant with the addition of 5-7 years of data. The basic pattern of our correlations with the sunspot cycle is shaped like a crescent with the largest correlations between $20^{\circ} \mathrm{N}$ and $45^{\circ} \mathrm{N}$ in the Pacific - Atlantic area, and it is present in every month. The correlations are statistically significant through most of the year, but the significance is especially high in summer and for the annual average. The statistically insignificant correlations in January-February improve when the data are grouped according to the wind near $50 \mathrm{mb}$ above the equator (the QBO). The east-wind years then show a highly significant pattern, the same as that in other months, whereas the west-wind years have no significant correlations outside the Arctic; there they are positive in contrast to the east years. Kodera (1993) has demonstrated that changes in the relationship between equatorial and polar regions on the decadal scale are a fundamental aspect of the opposite signs in the arctic correlations with the sunspot cycle in January-February.

Acknowledgments. We thank the members of the Stratospheric Research Group, Freie Universität Berlin, for technical and professional assistance. Part of the research was funded by the Bundesministerium für Forschung und Technologie, Projekt 07KF2012.

\section{REFERENCES}

Bottomley, M., Folland, C.K., Hsiung, J., Newell, R.E. \& Parker, D.E. 1990 Global Ocean Temperature Atlas (GOSTA). Joint Met. Office/MIT Project, Project supported by US Dept of Energy, US NSF and US Office of Nav. Res. 20+iv pp and 131 Plates, HMSO, London.

DAmeris, M. \& Ebel, A. 1990 The quasi-biennnial oscillation and major atmospheric warmings: a three-dimensional model study. Ann. Geophys. 8, 79-86.

Godson, W.L. 1991 The recognition and assessment of decadal-scale variation of the effects of solar variability on climate. J. Geomagn. Geoelectr. 43, 785-793.

Holton, J.R. \& TAN, H.CH. 1980 The influence of the equatorial QBO in the global circulation at 50mb. J. Atmos. Sci. 37, 2200-2208.

Holton, J.R. \& TAN, H.CH. 1982 The quasi-biennial oscillation in the northern hemisphere lower stratosphere. J. Met. Soc. Japan 60, 140-148. 
HOOD, L.L., JiRIKovic, J.L. \& MCCoRMACK, J.P. 1993 Quasi-Decadal variability of the stratosphere: Influence of long-term solar ultraviolet variations. J. Atmos. Sci. 50, in press.

KoderA, K. 1993 The quasi-decadal modulation of the influence of the equatorial QBO on the north-polar stratospheric temperatures. J. Geophys. Res. 98, 7245-7250.

LABITZKE, K. 1987 Sunspots, the QBO, and the stratospheric temperature in the North Polar region. Geophys. Res. Lett. 14, 535-537.

LABITZKe, K. \& VAN LOON, H. 1988 Association between the 11-year solar cycle, the QBO and the atmosphere. Part I: The troposphere and stratosphere in the Northern Hemisphere in winter. J. Atmos. Terr. Phys. 50, 197-206.

LABITZKe, K. \& VAN LoON, H. 1992 Associations between the 11-year solar cycle and the atmosphere. Part V: Summer. J. Clim. 5, 240-251.

LABITZKE, K. \& VAN LOON, H. 1993 Some recent studies of probable connections between solar and atmospheric variability. Ann. Geophysicae 11, in press.

LIVEZEY, R.E. \& Chen, W.Y. 1983 Statistical significance and its determination by Monte Carlo techniques, Mon. Weath. Rev. 111, 46-58.

SEllers, W.D. \& LIU, W. 1988 Temperature patterns and trends in the upper troposphere and lower stratosphere. J. Clim. 1, 573-581.

Shea, D.J., van Loon, H. \& Labitzke, K. 1992 Point correlations of geopotential height and temperature at $30 \mathrm{mb}$ and between $500 \mathrm{mb}$ and $30 \mathrm{mb}$. NCAR Techn. Note, NCAR/TN$368+$ STR.

Tinsley, B.A. \& DeEn, G.W. 1991 Apparent tropospheric response to MeV-GeV particle flux variations: A connection via electrofreezing of supercooled water in high-level clouds? J. Geophys. Res. 96, 22283.

VAN Loon, H. \& LABITzKe, K. 1990 Association between the 11-year solar cycle, the QBO and the atmosphere. Part IV: The Stratosphere, not grouped by the phase of the QBO. J. Clim. 3, 827-837. 